\title{
FACHADAS TRANSPARENTES: SISTEMAS ACTIVOS Y PASIVOS
}

\author{
RENATO D'ALENÇON | PROFESOR, TECHNISCHE \\ UNIVERSITÄT BERLIN \\ Arquitecto por la Pontificia Universidad Católica de Chile. Master en \\ Arquitectura (MArch) por la Universidad de Cornell, E.E.U.U. En 2002 \\ obtuvo una Beca Fulbright para sus estudios de Master y en 2007 una \\ beca de la Deutsche Akademischer Austausch Dienst DAAD para reali- \\ zar su investigación de doctorado en la Technische Universität Berlin. \\ Ha sido docente invitado en la Universidad de Chile y la TU-Berlin, \\ donde se desempeña actualmente.
}

\section{De la doble fachada vidriada a la integra- ción de sistemas mecánicos}

A partir de la década de 1990 aparece una nueva generación de fachadas acristaladas diseñadas para mediar entre las condiciones climáticas y los requerimientos de con-

fort. Las fachadas de dobles pieles ventiladas DPV -Double Skin Façades, DSF- surgieron entonces como una alternativa prometedora para abordar los problemas de los muroscortina, en particular el aislamiento acústico, la pérdida de calor y el sobrecalentamiento, mediante la combinación de un buffer acústico y térmico con el uso de protecciones solares dentro de la cámara vidriada. A partir de las DPV se desarrollaron otras tipologías que, aunque reciben nombres diversos, se pueden separar en dos grupos: fachadas inteligentes o responsivas, FIR -Intelligent or Responsive Façades, IRF-, aquellas que presentan la capacidad de adaptarse o responder a las condiciones del medio o del uso; y fachadas integradas avanzadas, FIA -Advanced Integrated Façades, AIF-, aquellas que incluyen instalaciones mecánicas y forman con ellas un sistema de acondicionamiento integrado.

El desarrollo inmediato de las envolventes vidriadas está relacionado con las innovaciones en ciertas tecnologías críticas en el mejoramiento de su desempeño ambiental, tales como: las propiedades de los materiales, los software de modelación, los sistemas de automatización, los sistemas de ventilación híbridos y los componentes de construcción reactivos. Estas tendencias sugieren que existe una amplia gama de alternativas para el desarrollo futuro de la integración en fachadas y de las estrategias pasivas y activas para la mediación de las condiciones climáticas y el confort. La innovación en las fachadas se implementa -como se discute más adelante- a partir de tipologías de fachadas existentes, a las que se agregan invenciones de manera incremental, por lo que resulta interesante hacer la distinción de estas tipologías y mostrar su desarrollo para comprender su potencial arquitectónico.
Dobles Pieles Ventiladas: Buffer termoacústico, protecciones y convección inducida en la cámara. Un sistema de fachada de doble piel ventilada DPV, consiste en una fachada con doble acristalamiento, es decir, un exterior en contacto con el medio ambiente y un interior en contacto con un recinto cerrado, separados por una cámara de aire cuyo espesor oscila entre los $15 \mathrm{~cm}$ y los $2 \mathrm{~m}$. El principio general es que la cámara puede ser usada alternadamente abierta - para mejorar la ventilación y proteger los recintos en veranoy cerrada -como colchón térmico en invierno, a través de diversas estrategias de ventilación, aislamiento y acceso-. La mayoría cuenta con protecciones solares situadas, a menudo, dentro de la cavidad para crear un efecto invernadero, aberturas en la piel externa e interna y, en algunos casos, ventiladores que permiten la ventilación forzada de la cavidad.

Los primeros edificios que incorporaron tales fachadas lo hicieron con fines de aislamiento acústico, pero su uso se multiplicó como un recurso para mejorar el aislamiento térmico en invierno y la ventilación en verano, a través de su control dentro de la cámara intermedia. Con la cámara cerrada en invierno estas soluciones alcanzan típicamente valores de entre 1,4 y $1,7 \mathrm{~W} / \mathrm{m}^{2} \mathrm{~K}$, normalmente con una combinación de doble vidriado hermético en la fachada y cristales simples en la hoja exterior.

\section{Cámara termo-acústica: OFICINAS HALLEN-} SEESTRASSE, LEON Y WOHLHAGE, BERLÍN, 1996 / Este edificio es otro de los primeros casos en incorporar DPV. Se encuentra situado en un sitio inmediato a una carretera urbana que se consideraba no edificable por razones de ruido (FIG.01). Para responder a las condiciones de implantación, el edificio se compone de un primer tramo de tres pisos de hormigón-altura de los muros de contención de la trinchera de la carretera-, donde se alojan estacionamientos y servicios, y un tramo superior de siete pisos de oficinas con fachadas de muro-cortina
(FIG.02). En la fachada sur, que enfrenta la carretera, se agrega una doble piel ventilada para el control de ruido y humos de combustión. Esta consta de dos hojas de cristal con una cámara de $85 \mathrm{~cm}$, con acristalamiento interior de doble vidriado hermético (WSG) y exterior con cristal de seguridad (ESG) reflectante, además de cortinas enrollables de protección solar en el interior de la cámara (FIG.03). No hay intercambio de aire directo al exterior sino a través de la cámara, que a su vez ventila con asistencia mecánica por medio de conductos de aire cerrados dentro de ella. La doble fachada es de tipo corredor, es decir, con particiones horizontales que dividen la cámara en cada piso, lo que impide el traspaso de ruido por vía aérea de un piso a otro, aun cuando los usuarios pueden abrir las ventanas para influir en el ambiente interior de forma individual o tener contacto con el exterior (FIG.04).

El objetivo original de la doble piel era reducir el impacto del ruido de la carretera. El sobrecalentamiento de la cámara -previsible dada la orientación sur- debía ser resuelto por el sistema de conductos mecánicos en su interior, puesto que la hoja exterior está sellada. Sin embargo, la cámara presenta altas temperaturas y es un problema para los usuarios abrir las ventanas a pesar de que son operables no solo en verano. El problema ha debido ser resuelto a través de la instalación de equipos de aire acondicionado en reemplazo de algunos de los radiadores de calefacción. Esta experiencia muestra que, incluso en un clima como el de Berlín de verano corto -julio y agosto- con temperaturas promedio cercanas a $18^{\circ} \mathrm{C}$ y extremas que raramente sobrepasan los $28^{\circ} \mathrm{C}$, el sobrecalentamiento por radiación solar se puede transformar en un problema de consideración.

Fachadas inteligentes o reactivas. Recopilación de información, modelación y control: Las fachadas inteligentes o reactivas -FIR- son aquellas que tienen la capacidad de 


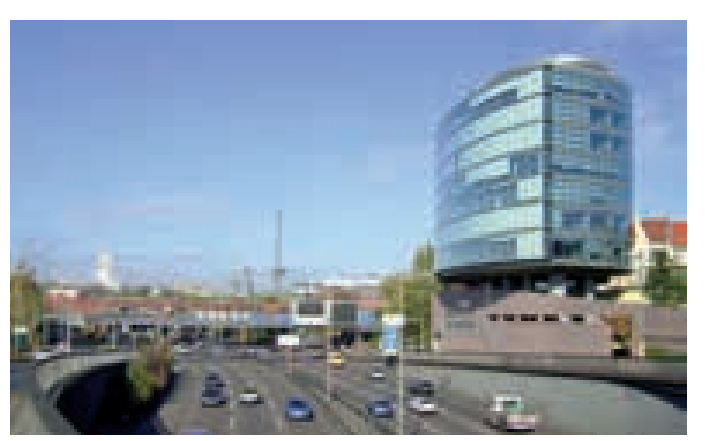

FIG.01>
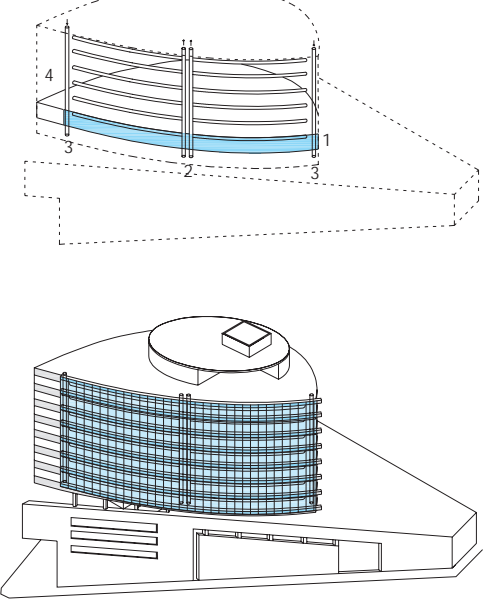

FIG.02 >

adaptarse a las condiciones del medio, gracias a un sistema que integra la recopilación de información sobre el desempeño del edificio, la modelación o evaluación de tal desempeño y los sistemas de control para corregirlo o adecuarlo a las condiciones del clima o del uso en un circuito permanente. Los elementos típicos que forman parte de estas estrategias son: regulación de la iluminación artificial y de la luz natural de acuerdo a las condiciones de nubosidad, y la regulación de calefacción o refrigeración según la carga de ocupación. Esencial para un edificio inteligente es la incorporación de un sistema de gestión del edificio -Building Management System, BMS-, una unidad central de procesamiento capaz de recopilar la información obtenida por medio de sensores - por ejemplo, una estación meteorológica en el edificio-, procesarla en un modelo ambiental, que incorpora otros datos, como los niveles actuales de ocupación -a menudo a través de un computador-, y controlar las respuestas que deben transmitirse a los dispositivos que regulan el funcionamiento de la fachada, por ejemplo, la apertura de ventanas para ventilación.

Normalmente, las medidas de control presentes están limitadas a algunos elementos o variables considerados relevantes en el diseño y están sujetas a una operación eficiente y bien informada. En muchos casos estos elementos se incorporan vistosamente
FIG.01 a 04 Edificio de oficinas en Hallenseestrasse. Leon y Wohlhage, Berlín, 1996. Cuerpo vidriado de oficinas con doble piel y cámara termo-acústica. Fuente: Fotografías de Renato D'Alençon y Alejandro Prieto; dibujos de Natalia Spörke

\section{FIG.02}

1. Doble fachada en corredor

2. Conductos de salida

3. Conductos de admisión

4. Fachada simple

S/E

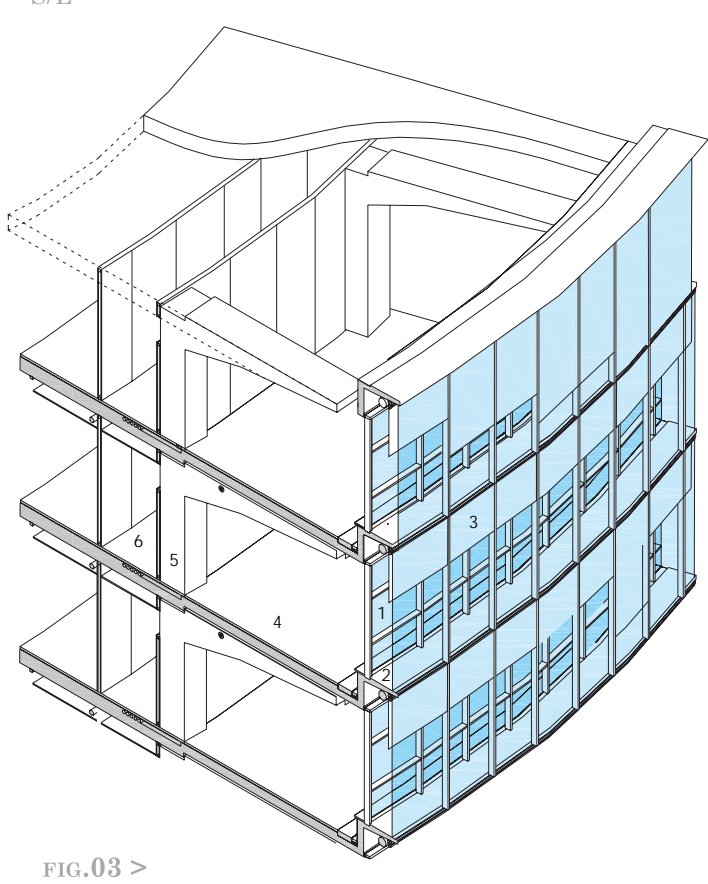

FIG.03 >

en las fachadas, lo que lleva a que se las defina como fachadas inteligentes. Sin embargo, las fachadas por sí solas no consiguen un edificio o una operación inteligente o reactiva a las condiciones del clima o la ocupación, sino que forman parte de un sistema integral del edificio en el que -como se ha dicho-, lo distintivo es la integración de evaluación, procesamiento y control. A continuación se presentan casos donde, a los elementos de protección a través de dobles pieles para mejorar el aislamiento térmico y reducir el sobrecalentamiento característico de las dobles fachadas, se suman sistemas de recopilación y procesamiento de información climática y de uso que, posteriormente, regulan la fachada con medidas como cierre de persianas para protección solar, aperturas de ventanas para ventilación y otros.

Fachada ventilada en cajón: RWE HEADQUARTERS, INGEHOVEN, OVERDIEK UND PARTNER, ESSEN, 1996 / En esta torre la forma cilíndrica responde a la búsqueda de una relación óptima entre el área de la piel exterior y el volumen, así como también respecto de la presión del viento y las pérdidas de calor. El acceso vertical es a través de una torre de ascensores fuera del volumen cilíndrico principal y los pisos se organizan en torno a dos núcleos de servicio interiores, con un corredor de acceso y una zona perimetral de oficinas (FIG.05). Como complemento a la doble fachada vidriada, el

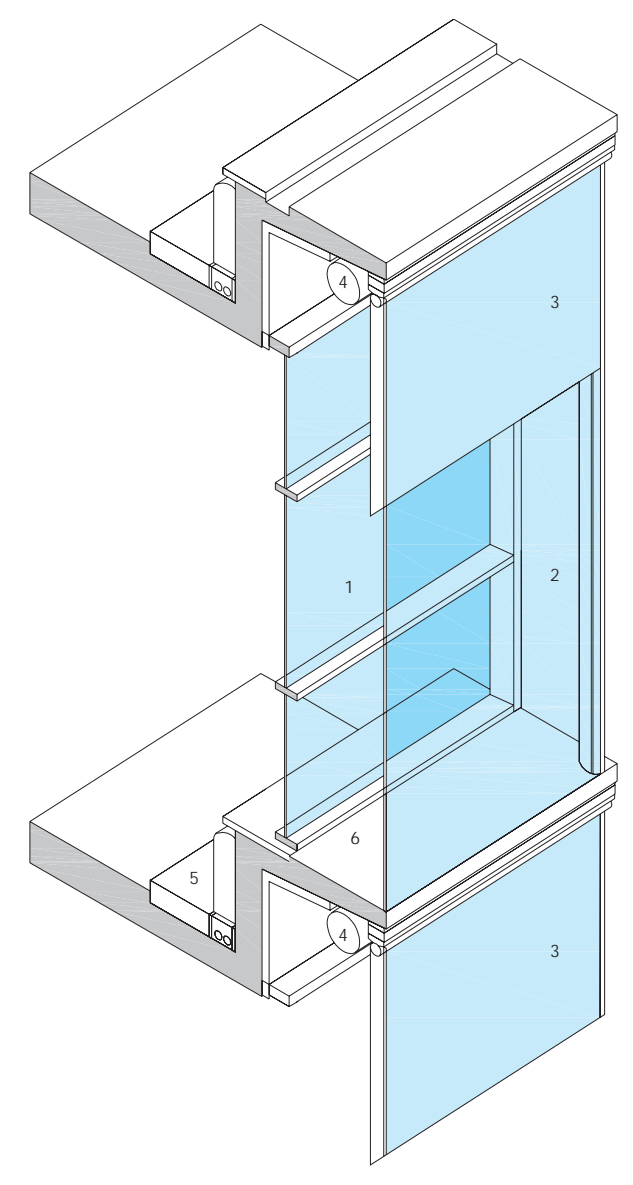

FIG.04 >

edificio cuenta con un sistema de ventilación centralizado en una planta técnica, ubicada en un piso intermedio, legible desde el exterior.

La doble fachada es de tipo cajón, con entrada y salida alternadas en un mismo elemento de fachada. Entrega a la cámara a través de rejillas de ventilación colocadas también alternadamente (FIG.05). Cada cajón de la doble fachada es una cámara de aire que permanece abierta al exterior a través de las admisiones mencionadas; por su parte, las ventanas se pueden abrir para permitir el control directo de la ventilación natural por parte del usuario, por sobre las condiciones del sistema mecánico del edificio (FIG.06). Cuando las condiciones meteorológicas impiden la apertura de las ventanas, la ventilación es proporcionada por una planta de aire acondicionado ubicada en un piso intermedio, con una capacidad mínima de intercambio de aire dimensionada a tal efecto. Las losas están expuestas como masa térmica a través de elementos modulares con láminas perforadas en el cielo, que no las separan de las oficinas (FIG.07).

Una ventaja del sistema de cajón, comprobada en este caso, es que la convección en el interior de la cámara es moderada gracias a que las ventanas correderas se pueden abrir para regular la circulación de aire con relativamente poco esfuerzo. Las dimensiones totales del edificio son también moderadas -28 pisos de altura y menos de 30 m de diámetro-, lo que 


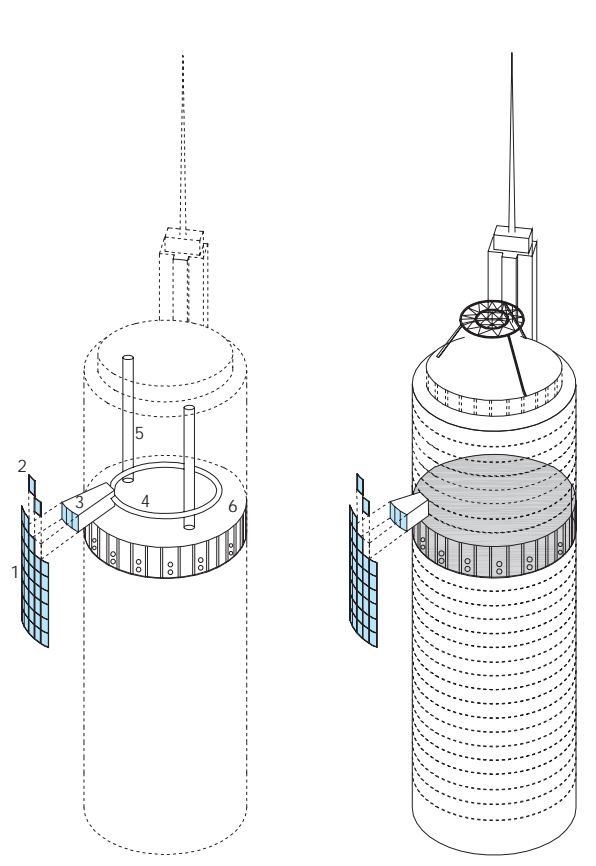

FIG.05 >
FIG.03

1. Hoia interior con ventanas operables

2. Voladizo antepecho

3. Hoja exterior con vidrios fijos y cortinas enrollables

4. Area de oficinas

5. Estructura de pilares y vigas en consola

6. Corredor de circulación

E.1/200

FIG. 04

1. Hoja interior con ventanas operables

2. Hoja exterior con vidrios fijos

3. Cortinas enrollables

4. Conducto de ventilación mecánica de la cámara

5. Rodapiê

6. Voladizo antepecho

E.1/50

FIG.05 a 07 Edificio de oficinas RWE. Ingehoven, Overdiek und Partner, Essen, 1996. Dobles módulos de fachada ventilados naturalmente y de forma separada; ventilación mecánica centralizada. Fuente: dibujos de Natalia Spörke

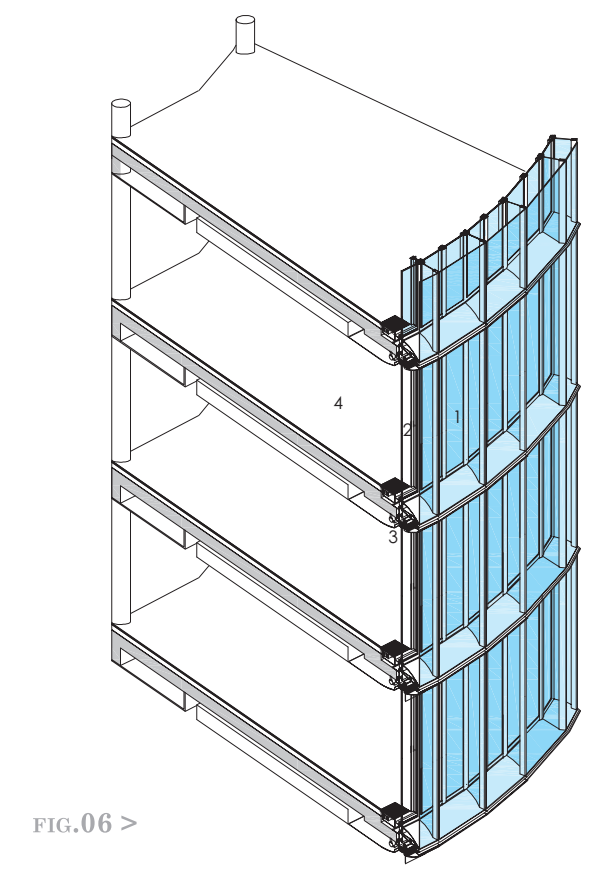

permite que la ventilación e iluminación de las fachadas sirva efectivamente a la superficie de la planta. Tanto la administración del edificio como los usuarios se muestran satisfechos por las condiciones de confort en el interior del recinto ${ }^{1}$. La fachada, a pesar de no tener elementos reactivos, forma parte de un sistema inteligente que se complementa con la masa de hormigón expuesta de las losas y el sistema de ventilación que se mantiene al mínimo gracias a la fachada.

AIF, integración de sistemas mecánicos en fachadas: las fachadas integradas avanzadas, FIA -Advanced Integrated Façades,
AIF - incluyen sistemas de acondicionamiento, como: la climatización descentralizada, la iluminación artificial y el cableado eléctrico. Knaack define una fachada integrada -Komponentenfassade - como aquella que incorpora "funciones tales como calefacción, refrigeración, ventilación, así como la dirección de la luz, la protección solar, la integración de la iluminación artificial e incluso la generación de energía con paneles fotovoltaicos"2 (Knaack, 2007).

Esta integración ofrece un número de ventajas potenciales, como por ejemplo ahorro de espacio en los conductos y las grandes unidades centralizadas, mejor adecuación de la toma de decisiones en la operación de los equipos a
IG.05

Doble fachada ventilada

2. Módulos de la fachada tipo cajón

3. Recinto de oficina servido por tres o más módulos

4. Conducto de distribución de la ventilación mecánica por piso

5. Conducto de ventilación vertical

6. Piso técnico intermedio de centrales de ventilación $\mathrm{S} / \mathrm{E}$

FIG.06

1. Hoja exterior doble fachada ventilada

2. Hoja interior doble fachada ventilada, con ventanas operables

3. Pieza de admisión y salida de ventilación de cada módulo al exterior

4. Recinto de oficinas servido por uno o más módulos E. $1 / 200$

FIG.07

1. Hoja exterior doble fachada ventilada

2. Hoja interior doble fachada ventilada, con ventanas operables

3. Pieza de admisión y salida de ventilación de cada módulo al exterior

4. Admisión al exterior

5. Salida al exterior

6. Rejilla de entrada a la cámara

7. Calefacción por radiadores bajo nivel de piso 8. Protecciones con persianas en el interior de la cámara E. $1 / 50$

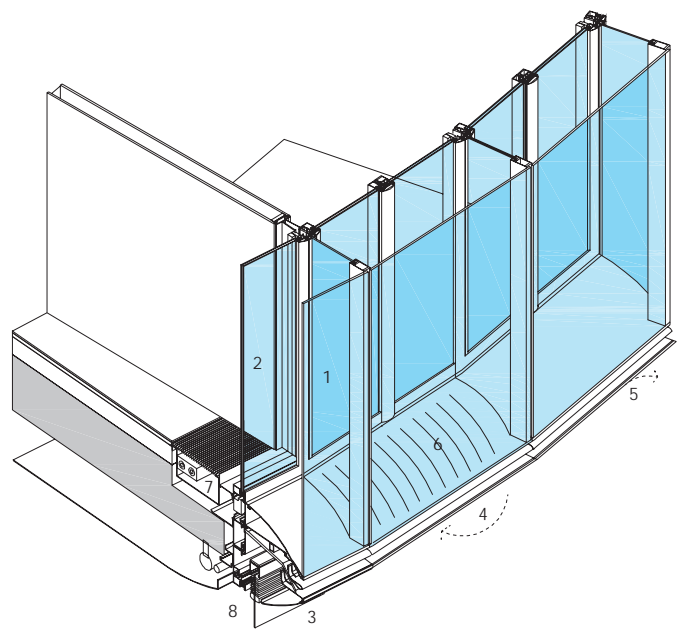

FIG.07 >

las necesidades de los usuarios y simplificación de los sistemas de mantenimiento mediante el uso de equipos estandarizados, de menor tamaño y más fácil acceso. Por otra parte, el riesgo es construir un edificio con un sistema de climatización convencional completo, con el costo añadido de una doble fachada.

A menudo estos sistemas se confunden con los sistemas pasivos de las dobles fachadas, pero son sustancialmente diferentes, ya que se basan en enfoques opuestos hacia los sistemas de energía. Mientras que las dobles fachadas se fundan en sistemas de energía pasiva y las fachadas integradas se complementan con un sistema mecánico o responden mecánicamente 
FIG.08 A 10 Edificio Capricorn. Gatermann - Schossig, Düsseldorf, 2005. Patios de ventilación vidriados y módulos de ventilación i-Modul Fuente: Fotografías de Renato D’Alençon; dibujos de Natalia Spörke

FIG.08

Patio vidriado semi-temperado

Fachada de un piso con módulos de ventilación i-Modul

Piso servido por el sistema de ventilación

4. Módulo i-Modul de ventilación descentralizada, integrada en la fachada y la ventana

$\mathrm{S} / \mathrm{H}$

a las condiciones de clima u ocupación; las fachadas integradas incorporan sistemas mecánicos en sí mismas y los integran en su funcionamiento.

\section{Módulo autónomo y envolvente hermé-} tica: CAPRICORN HOUSE, GATERMANN - SCHOSSIG DÜSSELDORF, 2005 / En este edificio se usó un módulo integrado, llamado i-Modul, desarrollado especialmente para constituir una fachada con componentes activos incorporados, en base a elementos preensamblados de 2,7 x 3,35 m. Complementariamente, cuatro grandes patios vidriados semi-temperados funcionan como espacios hacia los que algunas ventanas pueden abrir, lo que sustituye, hasta cierto punto, el acceso directo de los usuarios al exterior (FIG.08-09).

Los paneles son herméticos y la operación de las ventanas está restringida, lo que ofrece una combinación de calefacción, refrigeración y aireación por medio de una unidad de ventilación descentralizada. El diseño de la fachada incluye, en cada módulo, componentes transparentes y opacos, que combinan luz y visibilidad, y permiten además reducir las ganancias por radiación solar, si se compara con muros-cortina completamente vidriados. (FIG.10). La iluminación natural es obtenida a través de los paños vidriados o de la sección superior del panel, que también incluye un equipo de iluminación artificial. La unidad misma consta de dos compartimentos: una parte baja, que contempla la toma de aire exterior y las trampillas o dampers de aire; y una parte alta, con el módulo mecánico que cuenta con recuperación de calor.

Las unidades son controladas centralmente, pero pueden ser maniobradas manualmente y ofrecen la alternativa de operar de un modo balanceado, con tasas regulares de suministro de aire fresco fijado en $60 \mathrm{~m}^{3} / \mathrm{h}$. De este modo, el funcionamiento de la envolvente del edificio está completamente regulado a través de los módulos y se desempeña de manera descentralizada, aunque con control central. Sin embargo, no se integra ventilación natural en el diseño de la unidad de ventilación, sino solo un cristal dentro del módulo de fachada, con lo que la percepción del espacio exterior por los usuarios está siempre mediada por los equipos de ventilación o por los patios vidriados.
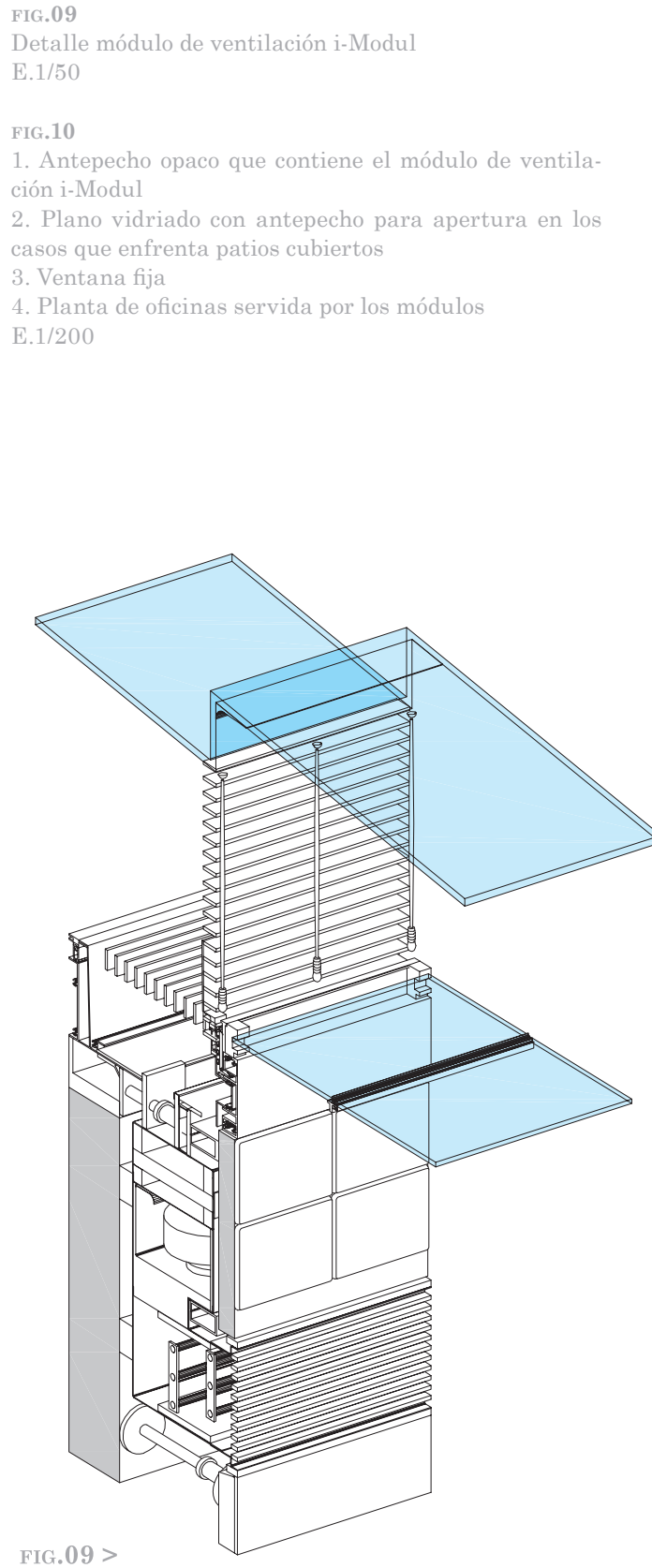

Fachada y sistema de ventilación descentralizado: POST TOWER, MURPHY Y JAHN, BONN, 2002 / En esta torre el aire puede ser templado en la cavidad de la doble fachada durante el invierno, o ventilado por convección durante el verano. Paneles móviles permiten la admisión de aire adicional en la cámara, que es más permeable que la DFV típica, lo que mejora su ventilación. Además el edificio puede ser aireado naturalmente, principalmente en primavera y otoño, y se apoya con el sistema mecánico en condiciones extremas del verano y del invierno (FIG.11). Después de ser templado en la cámara, el aire es tomado en las unidades descentralizadas de suministro de aire colocadas en el entrepiso, e inyectado en los espacios de trabajo. El aire que sale de las oficinas es llevado a los Sky Courts (FIG.12), que se utiliza para mantener espacios semitemperados antes de ser evacuado al exterior. Además de la ventilación, las unidades descentralizadas

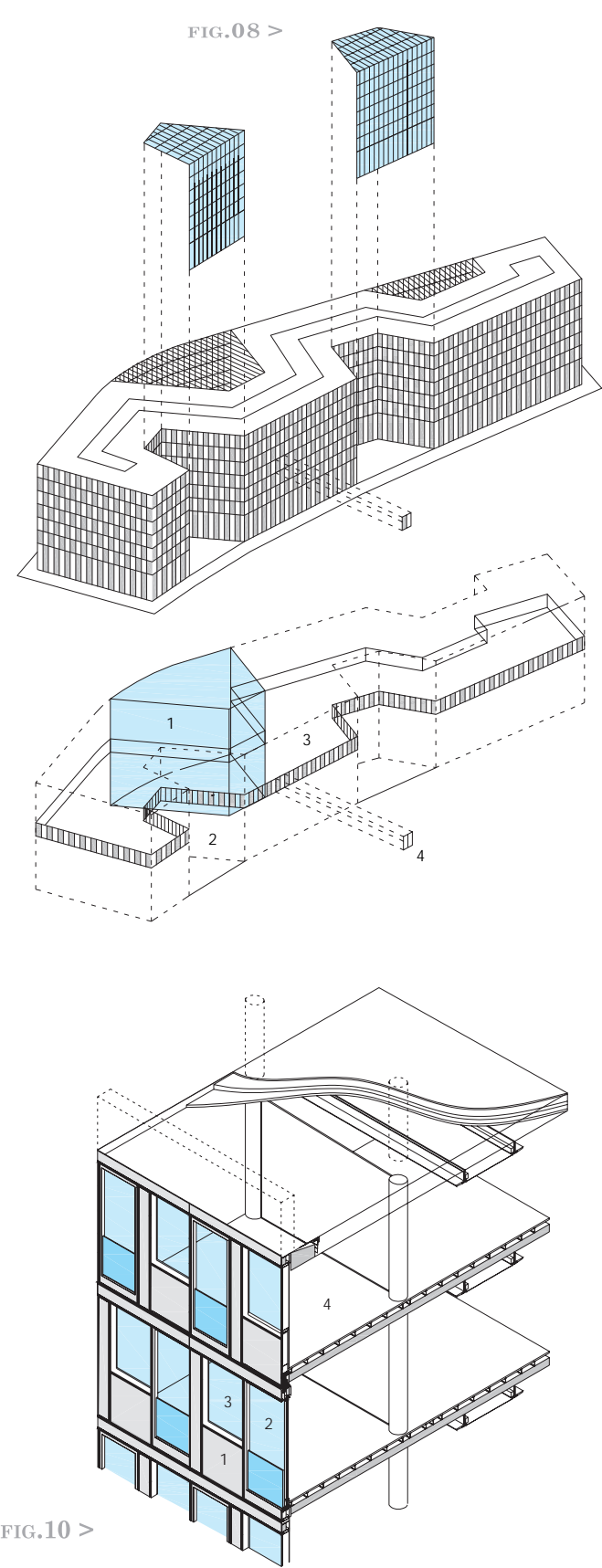

también regulan la temperatura del aire de admisión.

Las pruebas realizadas en modelos a escala real por el productor indican que las cargas de refrigeración y la demanda total de energía son más o menos comparables a las de un sistema central de climatización (FIG.13). En comparación, las ventajas son: menor consumo de energía para la distribución de la masa de aire acondicionado, reducción de los costos de construcción, instalación de equipos y mantenimiento simplificados, y control individual de las temperaturas y de las tasas de ventilación (Franze, et ál, 2003).

El enfoque de las DFV en el Post Tower es mixto, tanto desde el punto de vista de las $\mathrm{DFV}$, mediante la inclusión de nuevas posibilidades de ventilación con la piel exterior de lamas, como de la ventilación mecánica. Esto evita un sistema centralizado y se ahorra el espacio para las unidades centrales y los conductos en cada piso. 

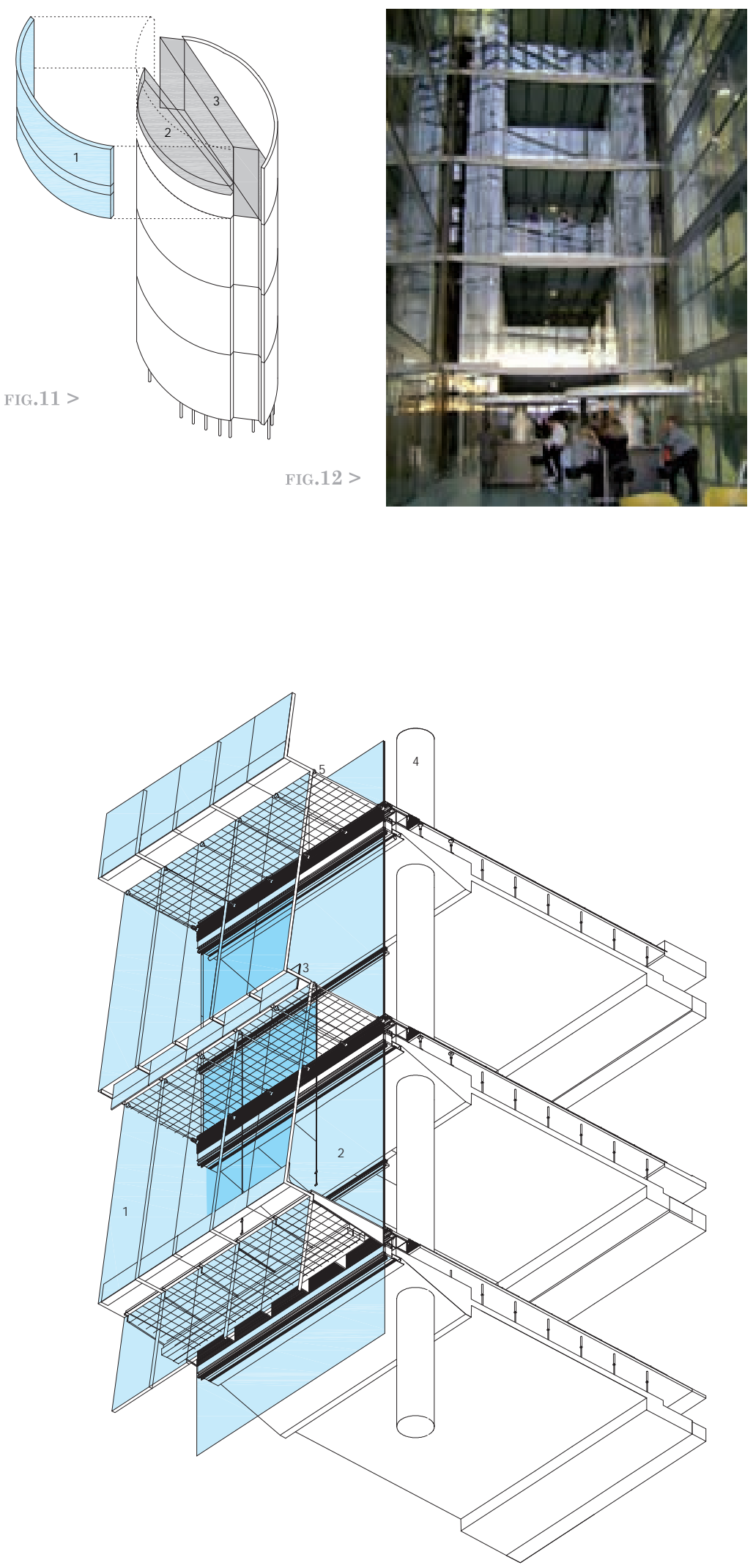

FIG.11 A 13 Edificio corporativo Deutsche FIG.13

Post. Murphy/Jahn, Bonn, 2002. Doble 1. Hoja exterior de la Doble Fachada Venfachada permeable en cada piso, con esco- tilad

tillas vidriadas horizontales; unidades de 2 . Hoja interior de doble vidriado, sin vanos sobrepiso de inyección de aire y patios semi- operables

temperados o Sky-Courts

Alejandro Prieto; dibujos de Natalia Spörke

FIG.11

1. Doble Fachada Ventilada de múltiple al-

1. Doble

tura (nueve pisos)

2. Piso ventilado desde la múltiple altura a

través de las unidades mecánicas en el piso

3. Sky-Court semi-temperado

$\mathrm{S} / \mathrm{E}$
3. Escotillas vidriadas operables para ventilación de la cámara

4. Unidad mecánica de ventilación en el piso falso

5. Piso de malla metálica para registro

E.1/100
CONCLUSIÓN

La oposición de métodos activos y pasivos se da por descontada, en la teoría y en la práctica. Sin embargo, edificios construidos recientemente dan cuenta de que existe una tendencia hacia la integración de sistemas mecánicos en las fachadas, que se suman a desarrollos anteriores como las dobles fachadas vidriadas.

Los casos de los edificios Capricorn y Post Tower muestran que, mediante la integración directa de los servicios de construcción en fachadas, es posible optimizar el sistema de acondicionamiento ambiental en un edificios luego de un estudio cuidadoso de sus fachadas. Estos sistemas ofrecen además otras ventajas, tales como: simplificar el mantenimiento, hacer menos invasivas las reparaciones y liberar espacio útil, lo que a su vez reduce las canalizaciones de aire acondicionado. El desarrollo en esta dirección es un camino de solución de los problemas de las fachadas vidriadas.

Los casos presentados se localizan en un clima relativamente frío, en que las ganancias solares en invierno son, en general, buscadas por razones de balance térmico y de percepción de los usuarios. A pesar de esto varios de ellos sufren de sobrecalentamiento en verano o de demandas de calefacción altas en comparación con fachadas convencionales. Este problema no es entonces propio de climas mediterráneos como el chileno -en el que, sin duda, adquiere mayor importancia-, que está aún por abordarse y resolverse, tanto en la investigación como en la práctica. + www.bestfacade.com/index.html, 2007 / BLÜMEL, E; KRAIL, J.; JÄHNIG, D. y W. wAGNER. Integrated Building Concepts - current IEA Trends and Monitoring Results. http://www.energytech.at/pdf/annex44_review-final.pdf/ compagno, Andrea. Intelligente Glasfassaden. Material, Anwendung, Gestaltung: Material, Practice, Design. Birkhäuser Verlag, Basilea, 1999. / foster, Norman. Norman Foster: Works. Editorial David Jenkins. Munich, 2003. / KNAACK, Ulrich; KLEIN, Tillmann; BILOW, Marcel y Thomas AUER. Façades: Principles of Construction. Birkhäuser Verlag, Basilea, 2007. / ochshorn, R. "Curtain Wall System”. Encyclopedia of 20th Century Architecture. Editorial R. Sennott, Nueva York, 2004. I PASQUAY, Till. "Natural ventilation in high-rise buildings with double facades, saving or waste of energy". Energy and Buildings 36, $N^{\circ}$ 4. Elsevier, Maryland, abril de 2004. / streicher, Wolfgang, et ál. "State of the Art". Bestfaçade: Best Practice for Double Skin Façades. WP 1 . http://www.bestfacade.com/pdf/downloads/ Lit database output.pdf, 2005. / wIGGINTON, Michael y Jude HARRIS. Intelligent skins. ButterworthHeinemann, Oxford, 2002. 